\title{
Carnets
}

Revue électronique d'études françaises de l'APEF

Deuxième série - 4 | 2015

Regards sur Camus

\section{Camus et sa politique du réel : une pensée pour l'Algérie}

João da Costa Domingues

\section{(2) OpenEdition}

1 Journals

Édition électronique

URL : https://journals.openedition.org/carnets/1503

DOI : 10.4000/carnets. 1503

ISSN : 1646-7698

Éditeur

APEF

Référence électronique

João da Costa Domingues, «Camus et sa politique du réel : une pensée pour l'Algérie », Carnets [En ligne], Deuxième série - 4 | 2015, mis en ligne le 30 mai 2015, consulté le 21 septembre 2021. URL : http://journals.openedition.org/carnets/1503; DOI : https://doi.org/10.4000/carnets.1503

Ce document a été généré automatiquement le 21 septembre 2021.

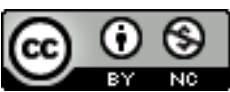

Carnets est mis à disposition selon les termes de la licence Creative Commons - Atribution - Pas d'utilisation commerciale 4.0 International. 


\title{
Camus et sa politique du réel : une pensée pour l'Algérie
}

\author{
João da Costa Domingues
}

1 «Je suis peut-être trop sensible au monde comme il va ${ }^{1}$, dit Camus. Cette déclaration « voltairienne $»^{2}$ à la fois de désolation et d'émerveillement face à la « bête humaine », il l'a vécue profondément et en a fait un de ses sujets d'élection. Pour Camus, l'homme reste l'unique valeur - hic et nunc, précisait Voltaire, l'homme ici et maintenant - à tel point que, comme il l'affirme dans L'Homme révolté, même « la (...) générosité envers l'avenir » consiste pour lui « à tout donner au présent » (Camus, 1965 : 707), et il ajoute :

Dans la lumière, le monde reste notre premier et notre dernier amour. Nos frères respirent sous le même ciel que nous, la justice est vivante. Alors naît la joie étrange qui aide à vivre et à mourir et que nous refuserons désormais de renvoyer à plus tard (Camus, 1965 : 708)

2 "J'ai mal à l'Algérie ", dit-il aussi, «j'ai mal à l'Algérie comme d'autres ont mal aux poumons » (Camus, 1965 : 963). Camus a toujours lutté pour l'éradication de la misère en Algérie, la suppression des grandes injustices et l'abolition des préjugés sociaux; mais non sans préciser que, pour y arriver, tous les moyens ne sont pas bons : et là réside toute sa pensée sociopolitique résumée à l'essentiel.

3 Camus a pensé l'homme, de manière indépendante mais pas neutre - la neutralité défendant, selon lui, à la fois le juste et l'injuste - alors même qu'il s'est toujours opposé à l'autorité qui opprime ${ }^{3}$, quelle qu'elle soit, et qu'il méprise, de toutes ses forces, le despotisme ${ }^{4}$. Qu'il parle de l'indigence matérielle de certains peuples, de la question sociale ou de la politique et de l'engagement, sa pensée émane toujours directement du quotidien de cet homo viator ${ }^{5}$ qui, tout pèlerin qu'il est, sait pourtant très bien qu'il chemine vers nulle part; un révolté, certes, mais qui, justement ${ }^{6}$ ne renonce pas. Et s'il est vrai que cette pensée concerne toute l'humanité, elle est pour Camus son propre étendard, car il ne renoncera jamais ni à la lutte contre la pauvreté, ni à la lutte sociale, ni à l'engagement de sa personne : « le malaise qui nous occupe, dit-il, est celui de toute une époque dont nous ne voulons pas nous séparer. Nous voulons penser et vivre dans notre histoire » (Camus, $1965: 312)$. 


\section{Camus et la pauvreté}

4 Une question concrète l'obsède : la fin de la misère arabe ; l'éradication de la famine en Algérie. Témoin et victime à la fois de cette misère dont souffre grand nombre d'habitants des quartiers algérois à l'époque, Camus estime qu'elle a exercé sur lui une grande influence, sur sa prise de conscience du monde en général et plus particulièrement de la condition humaine. Souvenons-nous que c'est au sein même de sa famille, dans le quartier de Belcourt, à Alger, qu'il a commencé sa vie au milieu de gens très humbles. Là justement qu'il a commencé à vivre, à penser et à chercher le sens de la vie. Cette quête, il ne l'abandonnera plus jamais et on pourrait même dire que c'est elle qui constitue le centre névralgique, à la fois de sa pensée, de son art et de sa vie : «J'aurais plaidé (...) pour que diminue dès maintenant l'atroce douleur des hommes » (Camus, 1965:363) ${ }^{7}$, a-t-il affirmé.

5 Pour remonter par ses écrits aux origines simples de Camus, il suffirait de le citer lorsqu'il nous livre, dans son roman inachevé Le Premier homme, le portrait de son père :

Un homme dur, amer, qui avait travaillé toute sa vie, avait tué sur commande, accepté tout ce qui ne pouvait s'éviter, mais qui, quelque part en lui-même, refusait d'être entamé. Un homme pauvre enfin. Car la pauvreté ne se choisit pas, mais elle peut se garder (Camus, $1994: 67$ ).

Orphelin de père depuis son plus jeune âge, élevé par sa mère, analphabète et presque sourde, qui doit gagner sa vie comme femme de ménage, ne pouvant par conséquent garantir que le strict minimum à la maison, c'est dans la pauvreté que Camus a appris une des plus grandes vertus humaines, à savoir la solidarité. Mais il n'en est pas dupe car il sait que cette condition l'a aussi limité dans ses rapports humains; elle l'a emprisonné dans un cercle réduit d'expériences et de rapports au monde. Il est d'ailleurs significatif que son passé miséreux lui revienne parfois à la mémoire comme une sorte d'excuse pour tout ce qui en lui ne correspond pas à ce dont il aspire ${ }^{8}$. Plus tard, dans son roman La peste , il nous dévoile combien ce dénuement matériel met à nu les illusions de l'existence et met à l'épreuve la force morale de chacun.

7 Par ailleurs, lorsqu'il écrit sur le peuple algérien, dans sa chronique sur la Kabylie, qui constitue son « voyage initiatique » dans le journalisme ${ }^{9}$, il y fait état de l'affreuse misère qu'il a pu y constater avec une lucidité dérangeante mais absolument réaliste :

Je crois pouvoir affirmer, dit-il, que $50 \%$ au moins de la population se nourrissent d'herbes et de racines et attendent pour le reste la charité administrative sous forme de distribution de grains. (...) sur 27000 kabyles (...) 10000 vivent dans l'indigence (...). C'est ce jour-là qu'on me fit voir la merveille de l'endroit: une vieille femme cassée en deux qui pesait 25 kilos. (...) Par un petit matin, j'ai vu à Tizi-Ouzou des enfants en loques disputer à des chiens (...) le contenu d'une poubelle (Camus, 1965 : 907-908).

8 Et on pourrait multiplier les exemples de cette longue déambulation à travers la souffrance du peuple Kabyle: des familles de dix enfants dont deux seulement ont survécu; des élèves qui dans les écoles s'évanouissent de faim, d'autres qui arrivent à l'école presque nus et couverts de poux (cf. Camus, 1965 : 908-909). Or, si, pour Camus, il existe dans tous ces malheurs une misère qui engendre un malaise tolérable, il y a aussi une misère qui, par l'absence complète de moyens pour satisfaire aux nécessités de subsistance, n'est pas une simple inégalité sociale, mais qui étouffe ses victimes, chaque individu, et l'anéantit, physiquement et moralement, lui enlevant toute capacité de 
s'exprimer et de se faire entendre. Et cette misère-là est, selon lui, un attentat à la dignité humaine.

\section{La question sociale}

9 Sensible à la cause de ceux qui souffrent de la faim, Camus l'est aussi à la cause sociale, et tout particulièrement aux victimes des injustices de toute sorte. Aussi ne doute-t-il jamais qu'il faille prendre parti et crier pour eux, à leur place. Lorsqu'il rédige sa chronique sur la Kabylie, ce n'est pas seulement la faim ou la misère matérielle des populations berbères indigènes de Kabylie qui l'occupe, mais bien toute la question sociale. Il y observe, par exemple, la distribution de grains à la population, action qu'il ne condamne pas, bien au contraire! Il considère néanmoins que les résultats sont presque nuls et qu'il faudrait par conséquent « lui préférer une politique sociale constructive » (Camus, 1965 : 912).

Sur la situation ouvrière, Camus écrit dans le journal Alger Républicain, du 8 juin 1939 :

On m'avait prévenu que les salaires étaient insuffisants. Je ne savais pas qu'ils étaient insultants. On m'avait dit que la journée de travail excédait la durée légale. J'ignorais qu'elle n'était pas loin de la doubler. Je ne voudrais pas hausser le ton, mais je suis forcé de dire ici que le régime du travail en Kabylie est un régime d'esclavage (Camus, 1965 : 915).

11 De plus, se penchant sur certaines initiatives des communes, pour contrer le chômage, communes "où les indigents exécutent des travaux d'utilité publique », ce qui ménage la dignité de la personne et lui permet de gagner un salaire, Camus soutient que, faute de pouvoir payer un salaire complet, ce ne sont que « des millions dépensés pour faire des ronds dans l'eau » (Camus, 1965 : 913); car, s'il est vrai que seul le travail permettrait à ceux qui meurent de faim de s'en sortir, il n'est pas moins vrai que ces initiatives, qui tout en les faisant travailler continuent à les laisser crever de faim, "constituent une exploitation intolérable du malheur » (Camus, 1965 : 913) ; les mots sont de Camus.

Enfin, sur l'enseignement apparemment subventionné et stimulé par la métropole, il le trouve mal adapté et ne correspondant pas aux vrais besoins des populations. En effet, alors que les Kabyles demandent des écoles pour leurs filles ${ }^{10}$, ce qui n'existe pas encore, et des écoles mieux adaptées aux populations - des écoles petites et proches de la population -, il ne voit que quelques "belles écoles» dans les grands centres, des " écoles-palais» (Camus, 1965 : 922) où sont dépensés des millions. Or ces majestueux exemples ne font que perpétuer les différences et le pouvoir de la métropole. Camus appelle cela « une politique qui consiste à donner une poupée de mille francs à un enfant qui n'a pas mangé depuis trois jours » (Camus, 1965 : 922).

En somme, ce reportage se fait bien l'écho de l'image du désarroi de ces populations kabyles qui ne se sentent plus chez elles. Et c'est de l'inhumanité que de faire perdurer cette situation, car c'est un peuple, dit Camus, « qui vit avec trois siècles de retard, et nous sommes les seuls à être insensibles à ce prodigieux décalage » (Camus, 1965 : 914). Pour y faire face, Camus prône l'action qu'il appelle la révolte, une révolte qui « ne peut se passer d'un étrange amour. Ceux qui ne trouvent de repos ni en Dieu ni en l'histoire se condamnent à vive pour ceux qui, comme eux, ne peuvent pas vivre : pour les humiliés " (Camus, 1965 : 707). 


\section{Entre pensée politique et engagement}

14 Camus n'a jamais renié ni ses origines plus que modestes ni son milieu pauvre; il y puise même sa volonté d'engagement, il leur doit aussi la formation de sa posture idéologique existentielle et moraliste surtout, car ne dit-il pas qu'il n'a pas appris «la liberté dans Marx », mais bien « dans la misère » (Actuelles III) ? Par conséquent, s'il peut à la rigueur se passer de systématisation d'une pensée politique stricte, il ne peut s'abstraire de l'engagement social ${ }^{11}$. Dans sa conférence du 14 décembre 1957 parlant de la condition de l'écrivain à l'époque, dans "L'artiste et son temps ", Camus est péremptoire : «Jusqu'à présent (...), l'abstention a toujours été possible dans l'histoire. (...) Aujourd'hui (...) le silence même prend un sens redoutable (...) » (Camus, $1965: 1079)$. Et il ajoute plus loin :

Bien entendu on peut toujours opposer à cet état de choses la lamentation humaniste (...). On peut aussi avoir des accès de tristesse civique. Mais cette tristesse ne change rien à la réalité. Il vaut mieux, selon moi, faire sa part à l'époque, puisqu'elle le réclame si fort (...) (Camus, 1965 : 1080).

Cette même réflexion s'applique parfaitement à Camus citoyen : l'engagement est pour lui un devoir, une obligation historique à laquelle il ne peut pas échapper puisqu'il ne peut pas se soustraire au monde qui l'entoure ; autrement il risquerait cette honte existentielle de ne pas avoir agi à temps, tel Clamence dans La Chute, tourmenté et honteux de ne pas avoir pu sauver la jeune femme qu'il a vue se jeter dans la Seine; hélas, elle ne se jettera pas une seconde fois! Ainsi dès lors que le monde nous impose un mal, on ne peut plus se détourner de l'histoire, et l'engagement devient un impératif pour tout un chacun, pour tout écrivain ou artiste : «Nous devons savoir (...) que nous ne pouvons pas nous évader de la misère commune, et que notre seule justification (...) est de parler, dans la mesure de nos moyens, pour ceux qui ne peuvent le faire » (Camus, $1965: 1092)$.

Par l'écriture ou par tout autre moyen, pour Camus, il est toujours question de réhabiliter l'individu dans son intégralité/dans son intégrité. Et si la charité occasionnelle ne suffit pas à contrer la misère, c'est qu'elle exige, au contraire, "une politique sociale constructive » (Camus, 1965 : 912). Car, offrir aux peuples la liberté sans la justice sociale ne ferait que perpétuer l'oppression : à quoi bon libérer un prisonnier en plein désert où il n'a aucune chance de survivre ? De même, offrir du pain sans la liberté ne serait qu'une insulte à la dignité de l'individu. C'est pourquoi Camus préférera toujours les valeurs qui embrassent la justice sociale et la liberté. Aussi, toujours engagé dans la lutte contre la misère matérielle du peuple arabe en général, il dénonce également une autre misère qui sévit au cœur de l'Algérie, le déplacement de milliers de gens éloignés de leur propre pays et séparés de leurs familles pour aller travailler et vivre dans des ghettos en banlieue, contre un salaire de misère, et dans les conditions effroyables que subit la nouvelle classe ouvrière.

Or, toutes ces observations sont on ne peut plus pertinentes et actuelles. Mais Camus n'a jamais dressé de plan politique proprement dit pour s'attaquer systématiquement à la question. On y trouve néanmoins une véritable réflexion sur les principes fondamentaux des sociétés, la première concernant justement les principes de liberté et de justice. Loin des idéologies qui promettent un monde parfait, Camus cherche à définir certaines conditions d'une pensée politique «délivrée de tout messianisme, et débarrassée de la nostalgie du paradis terrestre " comme il le dit dans "Ni Victimes ni Bourreaux" (Camus, 1965 : 335). Sans idéalisme, il voit bien que "pour être fécondes", justice et 
liberté « doivent trouver, l'une dans l'autre, leur limite" (Camus, 1965 : 694), autrement la liberté absolue se transformerait en droit du plus fort et la justice absolue anéantirait la liberté puisqu'elle supprimerait toute contradiction.

À la question qui se pose alors du rapport entre les moyens et la fin dans l'établissement, dans une société donnée, de ces principes, la réponse de Camus est sans ambages. En effet, si la question est de savoir « si nous avons le droit de tuer cet autre devant nous ou de consentir qu'il soit tué ", c'est-à-dire si la fin peut justifier n'importe quels moyens, sa réponse est catégorique : "[l]'action révoltée authentique ne consentira à s'armer que pour des institutions qui limitent la violence, non pour celles qui la codifient » (Camus, $1965: 695)$.

C'est une révolution - puisque c'est l'homme révolté qui parle ; mais il faut encore qu'elle soit efficace, c'est-à-dire réalisable et non pas idéale, sans être oppressive, autrement elle n'aura servi à rien. L'esprit révolutionnaire ne doit jamais se couper du réel, dit-il, tout en sachant que sa seule force réside dans le respect des moyens ${ }^{12}$.

Camus a donc bien prôné une politique non autoritaire qui protégerait tous les membres de la société contre l'injustice politique et économique et leur garantirait la liberté (cf. Letemendia : 2010). Pas une politique en tant que système concret - il ne l'a pas conçue comme système - mais plutôt comme réalisation d'une société qui apporterait non le bonheur, certes, mais les conditions indispensables pour l'atteindre ${ }^{13}$. En fait, il croit à l'amélioration obstinée, chaotique mais inlassable de la condition humaine (cf.Camus, 1965 : 311-316 $)^{14}$.

21 Après avoir longuement réfléchi non seulement sur les répressions aveugles, sur les crimes terroristes en Algérie, mais aussi sur la nécessité de faire droit aux revendications du peuple arabe concernant la réparation des mutilations infligées, et malgré toutes les différences entre les populations d'origine arabe et européenne, Camus aspire à une Algérie en cohabitation, multiethnique et multiculturelle ${ }^{15}$. Il rêve d'une Algérie où Français et Musulmans, tous Algériens, vivraient en paix, car, dit-il encore, « les Français d'Algérie ne sont pas tous des brutes assoiffés de sang, ni tous les Arabes des massacreurs maniaques " (Camus, 1965 : 971). Pour Camus, l'heure est au dialogue, à la prise de responsabilités des intervenants, sans exception, et au développement d'une politique adaptée à la circonstance de ce peuple maghrébin, où soient respectés les droits des Arabes mais aussi ceux des blancs. Or ceci ne constitue pas une «doctrine " politique, mais juste l'annonce de quelques préludes à toute politique future. Aux gouvernants, après, de choisir, selon le moment et la situation, la façon de régler les conflits, pourvu qu'ils préservent l'homme.

En définitive, qu'il nous parle de l'Algérie ou de la France ${ }^{16}$, plus qu'un système politique à proprement parler, Camus nous offre une vision de la lutte sociale. Homme passionné, homme sensible "au monde comme il va ", Camus est donc un social, et non un stratège politique ${ }^{17}$, un littéraire, rêveur parfois, non un économiste. Victime d'abord, puis témoin et citoyen engagé contre toute atteinte à la dignité humaine, il est partisan d'une " politique du réel », si bien expliquée dans L'homme révolté, laquelle se doit de résoudre les problèmes humains et atteindre des buts, limités certes, mais concrets, tels que l'éradication de la famine en Algérie, la fin de la misère arabe, l'arrêt de massacres d'innocents, l'instauration de la paix ${ }^{18}$; car tout compte fait - et il est admirable que l'on puisse trouver chez un auteur aussi pessimiste une expression aussi claire de la foi en l'homme dès 1947 -, «il y a dans les hommes plus de choses à admirer que de choses à mépriser» (Camus, $1962:$ 1473). 
mus préconise somme toute une société pensée pour l'humain, indépendamment du système politique qui lui donnerait corps et qu'il n'a, à ma connaissance, jamais précisé dans ses textes; une société fondée sur la justice et la liberté ${ }^{19}$; une société basée sur le principe essentiel que l'homme est la seule et unique valeur qui compte. Et s'il ne s'agit pas là d'une politique, est-ce que cela ne vaut pas mieux qu'une politique? C'est la révolution, comme il le dit dans Les Justes. "La révolution, bien sûr ! Mais la révolution pour la vie, pour donner une chance à la vie (...)» (Acte I). Enfin, une révolution qui ne prétend pas être définitive, puisque «tout effort humain est relatif. Nous croyons justement aux révolutions relatives » (Camus, 1965 : 1527), précise Camus. Une action située, donc, agissante bien que limitée, c'est-à-dire à mesure d'homme.

\section{BIBLIOGRAPHIE}

CAMus, Albert (1962-1965). CEuvres complètes. Paris : Gallimard, « Bibliothèque de la Pléiade », tomes I et II.

CAMus, Albert (1983). Euvres complètes. Paris : Gallimard, 6 vols.

CAMUS, Albert (1994). Le premier homme. Paris : Gallimard.

GUÉRIN, Jeanyves (dir.) (1986). Camus et la politique. Actes du colloque de Nanterre 5-7 juin 1985. Paris : L'Harmattan.

GuÉRIN, Jeanyves (dir.) (2009). Dictionnaire Albert Camus. Paris : Robert Laffont.

LETEMENDiA, Veronica C. (2010). «La pauvreté dans les écrits d'Albert Camus », Revue Quart Monde, $\mathrm{N}^{\circ} 215$ - Développement durable : avec ou sans misère?

LotTMAN, Herbert R. (1978). Albert Camus. Paris : Seuil.

\section{NOTES}

1. "Je suis peut-être trop sensible au monde comme il va. Mais finalement il n'est sans doute pas mauvais que quelques hommes au moins restent en alerte et ne puissent prendre leur parti de ce qui opprime ni, cette correspondance en est la preuve, de ce qui sépare " (Camus, 1965 : 1743). Sauf indication contraire, toutes les citations des œuvres de Camus seront faites à partir des deux tomes des Editions de la Pléiade.

2. Dans Zadig ou le monde comme il va, de Voltaire (1748), Babouc observe Persépolis (Paris) et est tantôt déchiré par la violence de cette ville, tantôt ébloui par la finesse de ses habitants.

3. Camus est très clair dans la négation des totalitarismes, aussi bien dans La Peste que dans L'État de Siège.

4. Dans Les justes, Kalliayev affirme : «J'aime la beauté, le bonheur ! C'est pour cela que je hais le despotisme » (Camus, $1965: 322$ ).

5. On dirait plutôt : l'homo socius - l'homme social, et pas à proprement parler viator - pèlerin, car s'il est vrai qu'il n'est pas d'ici - puisque ici il se sent étranger -, sa condition n'est pas non plus celle du pèlerin, puisqu'il ne va nulle part non plus! Camus croit pourtant à « l'amélioration 
obstinée, chaotique mais inlassable de la condition humaine » (Camus, 1965 : 311) ; et en ce sens, pour lui, la vie humaine ressemblerait à un vrai pèlerinage, toujours vers une condition meilleure.

6. Dans L'homme révolté, Camus explique sa vision de la lutte sociale et politique. «Qu'est-ce qu'un homme révolté ? Un homme qui dit non. Mais s'il refuse, il ne renonce pas : c'est aussi un homme qui dit oui, dès son premier mouvement " (Camus, 1965 : 423). «Le mal qui éprouvait un seul homme devient peste collective. Dans l'épreuve quotidienne qui est la nôtre, la révolte joue le même rôle que le cogito dans l'ordre de la pensée: elle est la première évidence. Mais cette évidence tire l'individu de la solitude. Elle est un lieu commun qui fonde sur tous les hommes la première valeur. Je me révolte, donc nous sommes » (Camus, 1965 : 432).

7. Albert Camus, « réponse à E. d'Astier » en 1948.

8. «C'est dans la pauvreté que j'ai trouvé et que je trouverai toujours les conditions nécessaires pour que ma culpabilité, si elle existe, ne soit pas honteuse du moins, et reste fière » (Camus, 1983, vol. VI : 288).

9. Camus aurait débuté dans le journalisme par le reportage, ayant travaillé comme rédacteurreporter à Alger Républicain en 1938.

10. «Le foyer, m'a dit l'un d'eux, n'est plus qu'un nom ou une armature sociale sans contenu vivant. Et nous éprouvons, tous les jours, l'impossibilité douloureuse de partager avec nos femmes un peu de nos sentiments. Donnez-nous des écoles de filles, sans quoi cette cassure déséquilibrera la vie des Kabyles » (Camus, 1965 : 921).

11. Aussi, s'il avait participé aux activités du Front Populaire - dans les années 35, et bien que très tôt inscrit dans les filières du PC, il en fut très vite exclu - entré en 34 il en sorti en 35 ( $c f$. Quilliot, in Guérin 1986 : 32) ou l'été 37, selon Lévi-Valensi (in Guérin, 1986 : 149) - et finit par se consacrer plutôt à la formation d'un groupe de théâtre. Camus est clairement dans la ligne des mouvements de défense de la culture plus que dans la politique.

12. «Mais l'esprit révolutionnaire, en Europe, peut aussi, pour la première et la dernière fois réfléchir sur ces principes, se demander quelle est la déviation qui l'égare dans la terreur et dans la guerre, et retrouver avec les raisons de sa révolte, sa fidélité » (Camus, 1965 : 696).

13. Le texte de Camus est tiré de Combat du 19 septembre 1944 : « Nous l'avons dit plusieurs fois, nous désirons la conciliation de la justice avec la liberté... Nous appelons justice un état social où chaque individu reçoit toutes ses chances au départ et où la majorité d'un pays n'est pas maintenue dans une condition indigne par une minorité de privilégiés... Et nous appellerons liberté un climat politique où la personne humaine est respectée dans ce qu'elle est comme dans ce qu'elle exprime... Notre idée est qu'il faut faire régner la justice sur le plan de l'économie et garantir la liberté sur le plan politique. Puisque nous en sommes aux affirmations élémentaires, nous dirons donc que nous désirons pour la France une économie collectiviste et une politique libérale... C'est dans cet équilibre constant et serré que résident non pas le bonheur humain qui est une autre affaire, mais les conditions nécessaires et suffisantes pour que chaque homme puisse être le seul responsable de son bonheur et de son destin » (Camus, 1965 : 1528).

14. Dans "Ni victimes ni bourreaux ", Camus présente la même idée, mais sous la forme d'un pari : « (...) j’ai toujours pensé que si l'homme qui espérait dans la condition humaine était un fou, celui qui désespérait des événements était un lâche. Et désormais, le seul honneur sera de tenir obstinément ce formidable pari qui décidera enfin si les paroles sont plus fortes que les balles » (Camus, $1965: 352$ ).

15. «Sur cette terre sont réunis un million de Français établis depuis un siècle, des millions de Musulmans, Arabes et Berbères, installés depuis des siècles, plusieurs communautés religieuses, fortes et vivantes. Ces hommes doivent vivre ensemble, à ce carrefour de routes et de races où l'histoire les a placés. Ils le peuvent, à la seule condition de faire quelques pas les uns au-devant des autres, dans une confrontation libre» (Camus, 1965 : 995). 
16. Il parle souvent en termes beaucoup plus larges, comme par exemple quand il affirme que «(...) la France et l'Europe ont aujourd'hui à créer une nouvelle civilisation ou à périr » (Camus, 1965 : 312).

17. Jeanyves Guérin remarque pourtant que, chez Camus, si les chroniques révèlent surtout le côté social, son œuvre théâtrale, romanesque ou philosophique ne nous livre pas moins une vision du monde, c'est-à-dire que l'œuvre est aussi politique ( $c f$. «Intellectuel », in Guérin, Jeanyves, $2008: 420$ ).

18. «Je parie pour la paix. C'est mon optimisme à moi. Mais il faut faire quelque chose pour elle et ce sera dur. C'est là mon pessimisme » (Camus, $1965: 384$ ).

19. Se justifiant l'une l'autre, et sachant que, pour y arriver, tous les moyens ne sont pas bons Les justes le disent de manière absolument claire.

\section{RÉSUMÉS}

«Trop sensible au monde comme il va ", Albert Camus penseur, écrivain et citoyen a toujours prôné l'éradication de la famine, des grandes injustices et des préjugés sociaux en Algérie. Cependant, pour y arriver, observe-t-il, tous les moyens ne sont pas bons, car le crime contre des innocents ne pourra jamais se justifier par le but à atteindre, aussi juste et noble qu'il soit. Né luimême dans la misère algéroise, il y a appris la valeur de la solidarité humaine. Révolté contre la condition humaine de son peuple algérien, il y a répondu par l'action de l'intellectuel écrivain et du citoyen engagé. Il a dénoncé aussi bien le travail précaire, "exploitation intolérable du malheur", que le manque d'écoles ajustées aux besoins des populations le plus démunies, s'engageant toujours dans la recherche de solutions réelles, par des actions simples, par des gestes à mesure d'homme.

"Too aware of the state of the world", Albert Camus, thinker, writer and citizen always fought to eradicate famine, great injustices and social prejudice in Argelia. However, to achieve those goals, as he himself points out, not all means are acceptable, since crime against innocent people will never be justified by its ends, regardless of how noble or how fair they may be. Born in the midst of Argiels' poverty, there he discovered the value of human solidarity. Angry, he reacted against the condition of his Argelian people as a writer and active citizen. Not only did he speak against precarious working conditions, "the exploitation of others' misery", but also against the lack of schools which cater for those most in need, committing himself to looking for real solutions, through simple and reasonable actions.

\section{INDEX}

Mots-clés : engagement, injustice, pauvreté, politique, préjugé, question sociale

Keywords : injustice, intervention, poverty, prejudice, social issues 


\section{AUTEUR}

\section{JOÃO DA COSTA DOMINGUES}

Un. de Coimbra - Centre de Littérature portugaise (CLP)

jcosta[at]fl.uc.pt 